Check for updates

Cite this: RSC Adv., 2018, 8, 33487

Received 5th July 2018

Accepted 23rd September 2018

DOI: $10.1039 / c 8 r a 05720 f$

rsc.li/rsc-advances

\title{
The tunable spin reorientation, temperature induced magnetization reversal, and spontaneous exchange bias effect of $\mathrm{Sm}_{0.7} \mathrm{Y}_{0.3} \mathrm{Cr}_{1_{-x}} \mathrm{Ga}_{x} \mathrm{O}_{3}$
}

\author{
Zhijie Ma, ${ }^{a}$ Guanghui Liu, ${ }^{a}$ Weijun Gao, ${ }^{a}$ Yuzhuang Liu, ${ }^{b}$ Liang Xie, ${ }^{c}$ Xuemin $\mathrm{He}^{a}{ }^{a}$ \\ Liqing Liu, ${ }^{a}$ Yongtao $\mathrm{Li}^{\mathrm{a}}$ and Hongguang Zhang (iD *ab
}

\begin{abstract}
In this work, we investigated the temperature dependent magnetic properties of $\mathrm{SmCrO}_{3}$ by codoping nonmagnetic ions at $\mathrm{Sm}$ - and $\mathrm{Cr}$-sites. The spin reorientation from $\Gamma_{4}$ to $\Gamma_{2}$ is tuned and the transition temperature $T_{\mathrm{SR}}$ is improved dramatically to near the liquid nitrogen temperature by $\mathrm{Ga}$ ion doping, which would be helpful to achieve its application in temperature sensitive spintronic devices and magnetic switching devices. An intrinsic temperature induced magnetization reversal effect from positive to negative under zero-field-cooling conditions is induced as well and its reversal evolution is strongly dependent upon doping. Moreover, the zero-field-cooling exchange bias effect still exists and shows a positive exchange bias field although it is suppressed with increase of doping concentration. Under the influence of doping nonmagnetic ions, lattice distortion is induced to some extent and the magnetic interactions of $\mathrm{Cr}-\mathrm{Cr}$ and $\mathrm{Sm}-\mathrm{Cr}$ are predominantly diluted, realizing control of the above phenomena. Those phenomena are discussed and successfully explained by considering the magnetic exchange interaction competitions including the isotropic, antisymmetric (or Dzyaloshinskii-Moriya interaction), and anisotropic superexchange interactions.
\end{abstract}

\section{Introduction}

Orthochromites $\mathrm{RCrO}_{3}(\mathrm{R}=$ rare-earth) have received great attention recently due to their possessing abundant magnetic effects (such as temperature induced magnetization reversal (TIMR), magnetic exchange bias (EB) effect, and spin reorientation (SR) phase transition), and ferroelectricity, making them a potential candidate for technological applications. ${ }^{1-7}$ Orthochromites $\mathrm{RCrO}_{3}$ have a distorted orthorhombic perovskite structure (Pbnm space group) with a canted antiferromagnetic structure below the Neel temperature $\left(T_{\mathrm{N}}\right)$ caused by Dzyaloshinskii-Moriya (DM) exchange coupling between the $\mathrm{Cr}$ ions. Three types of antiferromagnetic configurations generally exist in orthochromites, noted in Bertaut notations ${ }^{8}$ as $\Gamma_{1}\left(A_{x}, G_{y}\right.$, $\left.C_{z}, C_{z}^{\mathrm{R}}\right), \Gamma_{2}\left(F_{x}, C_{y}, G_{z}, F_{x}^{\mathrm{R}}, C_{y}^{\mathrm{R}}\right)$, and $\Gamma_{4}\left(G_{x}, A_{y}, F_{z}, F_{x}^{\mathrm{R}}\right)$. The complex magnetic interactions of $\mathrm{Cr}-\mathrm{Cr}, \mathrm{Cr}-\mathrm{R}$, and $\mathrm{R}-\mathrm{R}$ in $\mathrm{RCrO}_{3}$, including the isotropic, symmetric, and antisymmetric anisotropic exchange interactions, result in an abundant magnetic phase diagram, such as, multiferroics, ${ }^{\mathbf{4 , 9}}$ multiple phase

${ }^{a}$ New Energy Technology Engineering Laboratory of Jiangsu Province, College of Science, Nanjing University of Posts and Telecommunications, Nanjing 210023, P. R. China.E-mail: hgzhang_njupt@hotmail.com

${ }^{b}$ College of Electronic and Optical Engineering \& College of Microelectronics, Nanjing University of Posts and Telecommunications, Nanjing 210023, P. R. China

${ }^{c}$ Department of Physics, North China University of Technology, Beijing 100144, P. R. China transitions, ${ }^{10,11}$ a magnetic glassy phase, ${ }^{12}$ TIMR, ${ }^{13-15}$ conventional $\mathrm{EB}^{6}$ and zero-field-cooling EB (ZEB) effects, ${ }^{\mathbf{1 4 , 1 6}}$ and the SR transition. ${ }^{17}$

One of the most interesting examples of this family is the newly emerging $\mathrm{SmCrO}_{3}$ which possesses two magnetic species, $\mathrm{Sm}$ and $\mathrm{Cr}$ ions. $\mathrm{SmCrO}_{3}$ is reported to be ordered in $\Gamma_{4}$ configuration below $T_{\mathrm{N}}$ at $191 \mathrm{~K}$ and exhibits a SR transition to $\Gamma_{2}$ configuration at $34 \mathrm{~K}^{\mathbf{4 , 1 8}}$ There is controversy about the SR transition so far. Gorodetsky et al. ${ }^{19}$ reported that below the SR transition temperature $\left(T_{\mathrm{SR}}\right)$ the magnetic structure of $\mathrm{SmCrO}_{3}$ changes from $\Gamma_{4}$ to $\Gamma_{2}$ continuously, making a second order transition. Most of the reports suggest that the second-order transition is attributed to a continuous rotation of $\mathrm{Cr}^{3+}$ moments. ${ }^{17,20}$ There are a few factors that give rise to the second order SR transition, for examples, the antisymmetric exchange interaction between $\mathrm{Cr}^{3+}$ and $\mathrm{Sm}^{3+},{ }^{6}$ and the exchange splitting and the t-e orbital hybridization between $\mathrm{Cr}^{3+}$ and $\mathrm{Cr}^{3+}$ ions. ${ }^{17}$ However, Tripathi et al. ${ }^{12}$ suggested that the SR transition in $\mathrm{SmCrO}_{3}$ would be a first order Morin type SR transition based on the analysis of the existence of phase coexistence and magnetic glass like freezing across $T_{\mathrm{SR}}$, the reason of which is due to the discrete flipping of $\mathrm{Cr}^{3+}$ ions from the high temperature $\Gamma_{4}$ to low temperature $\Gamma_{1}$ configuration. Very recently, Tripathi et al. ${ }^{21}$ investigated the thermal evolution of magnetic configuration in $\mathrm{SmCrO}_{3}$ by neutron diffraction and magnetometric study and confirmed that the uncompensated canted antiferromagnetic structure $\Gamma_{4}$ occurs below $T_{\mathrm{N}}$, the collinear 
antiferromagnetic structure $\Gamma_{1}$ occurs below $10 \mathrm{~K}$, and a nonequilibrium configuration with co-occurring $\Gamma_{1}$ and $\Gamma_{4}$ phases occurs at $10 \mathrm{~K} \leq T \leq 40 \mathrm{~K}$. The competition between magnetocrystalline anisotropy and free energy derived from isotropic and antisymmetric exchange interactions among different pairs of magnetic ions is observed to govern the mechanism of SR effect. $^{21}$ Therefore, the investigation of the SR transition, especially from the view of artificial control of the exchange interactions between chromium and samarium ions, is full of interest and finding its tunable factors and achieving its control will benefit for applications in thermomagnetic power generation, ultrafast spin switching. ${ }^{22,23}$

Moreover, the complex magnetic exchange interactions of $\mathrm{Sm}-\mathrm{Cr}$ or/and $\mathrm{Cr}-\mathrm{Cr}$ and their competitions are mostly considered to be the origin of the TIMR and conventional EB and ZEB effects as well. There was no negative magnetization originally in $\mathrm{SmCrO}_{3}$ itself and the TIMR effect can be induced by transitional metal ions doping, such as, Fe, ${ }^{10,24}$ and $\mathrm{Mn}^{\mathbf{1 3 , 2 5}}$ Nevertheless, negative magnetization in $\mathrm{SmCrO}_{3}$ will be outstanding due to the intrinsic strong coupling between $\mathrm{Sm}^{3+}$ and $\mathrm{Cr}^{3+}$ spins sublattices, ${ }^{18}$ which would also be responsible for the existence of EB effect. Due to the coupling between Sm and $\mathrm{Cr}$ ions, a ZEB effect appears in $\mathrm{SmCrO}_{3}{ }^{18,20}$ And nonmagnetic rare-earth ions doping at Sm-site confirm the influence of $\mathrm{Sm}^{3+}$ on the ZEB effect, ${ }^{\mathbf{1 4 , 2 0}}$ which would be of great interest to electric field control of EB devices as it eliminates the requirement of external magnetic field to create the unidirectional anisotropy.

In this work, we investigated the temperature dependent magnetic properties of $\mathrm{SmCrO}_{3}$ by codoping nonmagnetic ions $\left(\mathrm{Y}^{3+}\right.$ and $\left.\mathrm{Ga}^{3+}\right)$ at Sm- and Cr-sites, respectively. The SR transition from $\Gamma_{4}$ to $\Gamma_{2}$ is tuned and the transition temperature $T_{\mathrm{SR}}$ is improved dramatically to the liquid nitrogen temperature by $\mathrm{Ga}$ ions doping. The outstanding intrinsic TIMR effect from positive to negative with temperature decreasing under zero-fieldcooling (ZFC) condition are induced as well at an appropriate doping content. Meanwhile, noting that a positive ZEB effect occurs at $5 \mathrm{~K}$ and is suppressed by doping and increasing of temperature. Those phenomena are mainly considered to be the competitions among the isotropic exchange interaction of $\mathrm{Cr}^{3+}$, the antisymmetric exchange interaction of $\mathrm{Cr}^{3+}$, the DM interaction, the single-ion magnetocrystalline anisotropy, and the isotropic and antisymmetric interaction of $\mathrm{Sm}$ and $\mathrm{Cr}$. The dilution of $\mathrm{Cr}-\mathrm{Cr}$ coupling by doping will highlight the $\mathrm{Sm}-\mathrm{Cr}$ coupling, leading to the significant change in SR, TIMR and ZEB effect.

\section{Experimental}

Polycrystalline samples of $\mathrm{Sm}_{0.7} \mathrm{Y}_{0.3} \mathrm{Cr}_{1-x} \mathrm{Ga}_{x} \mathrm{O}_{3}(x=0,0.1,0.2$, and 0.3 ) were prepared by conventional solid state reaction method. ${ }^{14,15}$ High purity (99.9\%) oxide of samarium $\left(\mathrm{Sm}_{2} \mathrm{O}_{3}\right)$, yttrium $\left(\mathrm{Y}_{2} \mathrm{O}_{3}\right)$, gallium $\left(\mathrm{Ga}_{2} \mathrm{O}_{3}\right)$ and chromium $\left(\mathrm{Cr}_{2} \mathrm{O}_{3}\right)$ were weighed and mixed at a stoichiometric ratio. The mixtures were first calcined at $1200{ }^{\circ} \mathrm{C}$ for $12 \mathrm{~h}$ with the heating rate of $2{ }^{\circ} \mathrm{C} \min ^{-1}$ and cooled down with the furnace. Finally, after regrinding and tableting, the resulting products were sintered at $1400{ }^{\circ} \mathrm{C}$ for $12 \mathrm{~h}$ with the same heating rate. The samples were obtained after cooling with the furnace.

The X-ray diffraction (XRD) measurement was performed by Bruker D8 diffractometer with $\mathrm{Cu} \mathrm{K} \alpha$ radiation at room temperature. The working current and voltage were $40 \mathrm{~mA}$ and $40 \mathrm{kV}$ respectively and the diffraction angle ranges from 20-70 degree with step of 0.02 degree. And the XRD patterns were refined by Rietveld method using PC-GSAS and EXPGUI programs. ${ }^{26}$ Measurement of Raman spectra of all samples with a range from 100 to $600 \mathrm{~cm}^{-1}$ were conducted by EZRaman-M Portable Raman System with $532 \mathrm{~nm}$ excitation wavelength $\mathrm{He}-\mathrm{Ne}$ laser. The field-cooling (FC) and $\mathrm{ZFC}$ temperature dependent magnetization curves of $\mathrm{Sm}_{0.7} \mathrm{Y}_{0.3} \mathrm{Ga}_{x} \mathrm{Cr}_{1-x} \mathrm{O}_{3}(x=$ $0.1,0.2,0.3)$ samples were measured at the temperature range of $5 \mathrm{~K}$ to $300 \mathrm{~K}$ under the magnetic field $H=100$ Oe by a superconducting quantum interference device (MPMS-XL-7). The ptype magnetic hysteresis data $(M-H$ loop) were recorded at constant $5 \mathrm{~K}$ in ZFC mode for $\mathrm{Sm}_{0.7} \mathrm{Y}_{0.3} \mathrm{Ga}_{x} \mathrm{Cr}_{1-x} \mathrm{O}_{3}(x=0.1,0.2$, 0.3 ) and at various temperatures 5,35 and $100 \mathrm{~K}$ for $x=0.1$, at 5 , 45 and $100 \mathrm{~K}$ for $x=0.2$, and at 5, 50 and $100 \mathrm{~K}$ for $x=0.3$, respectively.

\section{Results and discussion}

Fig. 1(a-c) shows the XRD patterns of $\mathrm{Sm}_{0.7} \mathrm{Y}_{0.3} \mathrm{Cr}_{1-x} \mathrm{Ga}_{x} \mathrm{O}_{3}(x=$ $0,0.1,0.2$ and 0.3 ) samples. The samples are regarded to be desired pure phase polycrystalline. Rietveld refinement results reveal that samples are the distorted orthorhombic perovskite structure with Pbnm (no. 62) space group, consistent with the parent sample $\mathrm{SmCrO}_{3} \cdot{ }^{\mathbf{6}, \mathbf{9}, 14}$ From Rietveld refinement results, we obtained data of lattice parameters $a, b, c$, cell volume of samples, the angle of out-of-plane $\mathrm{Cr}-\mathrm{O}_{1}-\mathrm{Cr}$, and the bond
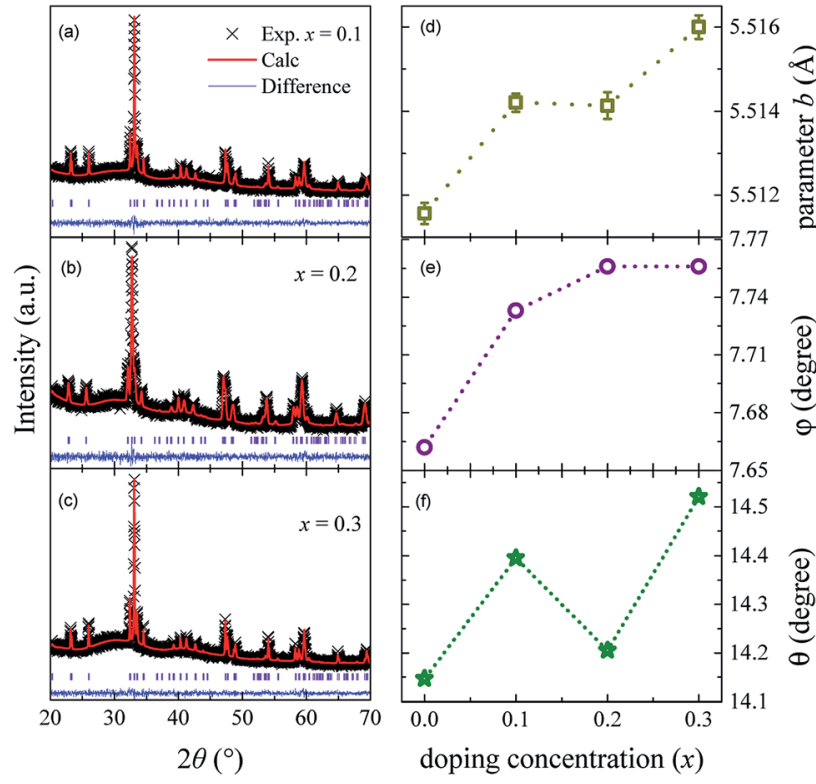

Fig. $1(a-c)$ The refined XRD patterns of $\mathrm{Sm}_{0.7} \mathrm{Y}_{0.3} \mathrm{Cr}_{1-x} \mathrm{Ga}_{x} \mathrm{O}_{3}(x=0.1$, 0.2 , and 0.3 ) samples. ( $d-f)$ The parameter $b$, the tilting angle $\varphi$ and rotational angle $\theta$ of $\mathrm{CrO}_{6}$ octahedral with doping concentration. 
Table 1 The parameters $a, b$, and $c$, cell volume $V$, the bond angle $\left(^{\circ}\right)$ of out-of-plane $\mathrm{Cr}-\mathrm{O}_{1}-\mathrm{Cr}$, the bond length of out-of-plane $\mathrm{Cr}$ - $\mathrm{O}_{1}(\AA)$, the rotational angle $\theta$ and the tilting angle $\varphi$ of $\mathrm{CrO}_{6}$, the compensation temperature $T_{\text {comp }}(\mathrm{K})$, the Neel temperature $T_{\mathrm{N}}$, the SR transition temperature $T_{\mathrm{SR}}$, and the characteristic temperature $\left(T^{\prime}\right)$ of Sm moment ordering, and the Curie constant $C\left(\mathrm{emu} \mathrm{K} \mathrm{mol}^{-1} \cdot \mathrm{Oe}^{-1}\right)$, the Weiss temperature $\Theta$, the fitted effective magnetic moment $\mu_{\text {eff }}$ by Curie-Weiss law and the theoretically calculated effective magnetic moment $\mu_{\text {eff }}^{*}$ by eqn (2), and the coupling coefficient $\alpha$ from eqn (3)

\begin{tabular}{lllll}
\hline Sample & $x=0$ & $x=0.1$ & $x=0.2$ & $x=0.3$ \\
$a(\AA)$ & $5.34442 \pm 0.00030$ & $5.34109 \pm 0.00029$ & $5.34554 \pm 0.00035$ & $5.33979 \pm 0.00029$ \\
$b(\AA)$ & $5.51157 \pm 0.00025$ & $5.51421 \pm 0.00022$ & $5.51414 \pm 0.00032$ & $5.51600 \pm 0.00028$ \\
$c(\AA)$ & $7.62624 \pm 0.00037$ & $7.62276 \pm 0.00032$ & $7.62916 \pm 0.00039$ & $7.62133 \pm 0.00045$ \\
$V\left(\AA^{3}\right)$ & $224.63 \pm 0.019$ & $224.50 \pm 0.018$ & $224.88 \pm 0.023$ & $224.48 \pm 0.021$ \\
$\mathrm{Cr}-\mathrm{O}_{1}-\mathrm{Cr}$ & $156.777(2)$ & $153.090(1)$ & $154.956(2)$ & $149.456(1)$ \\
$\mathrm{Cr} / \mathrm{Ga}-\mathrm{O}_{1}$ & $1.97642(2)$ & $1.97336(5)$ & $2.00591(5)$ & $1.98948(1)$ \\
$\chi^{2}$ & 1.097 & 1.096 & 1.073 & 1.091 \\
$\Theta$ & $14.14676 \pm 0.00028$ & $14.39503 \pm 0.00026$ & $14.20497 \pm 0.00035$ & $7.752124 \pm 0.00029$ \\
$\Phi$ & $7.66203 \pm 0.00078$ & $7.73276 \pm 0.00071$ & $7.73547 \pm 0.00086$ & $75627 \pm 0.00083$ \\
$T_{\text {comp }}$ & - & 163 & 74 & 134 \\
$T_{\mathrm{N}}(\mathrm{K})$ & 178 & 152 & 58 & 73 \\
$T_{\mathrm{SR}}(\mathrm{K})$ & 28 & 43 & 21 & 21 \\
$T^{\prime}(\mathrm{K})$ & - & 21 & $3.054 \pm 0.011$ & $-549.6 \pm 2.1$ \\
$C$ & $3.465 \pm 0.014$ & $2.674 \pm 0.012$ & 4.94 & $-483.1 \pm 1.4$ \\
$\Theta(\mathrm{K})$ & $-748.4 \pm 3.1$ & $-419.8 \pm 2.1$ & 3.53 & \\
$\mu_{\text {eff }}\left(\mu_{\mathrm{B}}\right)$ & 5.27 & 4.63 & & 3.87 \\
$\mu_{\mathrm{eff}}^{*}\left(\mu_{\mathrm{B}}\right)$ & 3.94 & 3.74 & &
\end{tabular}

length of out-of-plane $\mathrm{Cr}-\mathrm{O}_{1}$, listed in Table 1 . It is noticeable that those parameters are not dramatically changed. Lattice parameters $a$ and $c$ both have the tendency to decrease with increasing doping concentration $x$, while parameter $b$ almost monotonically increases. This results from the fact that the radius of $\mathrm{Ga}^{3+}(0.620 \AA)$ ion is a bit larger than that of $\mathrm{Cr}^{3+}(0.615$ $\AA)$. With an incorporation of $\mathrm{Ga}$ ions, the degree of crystal structure distortion to some extent increases as well, which leads to the increment of the $\mathrm{Cr}(\mathrm{Ga})-\mathrm{O}$ bond length and $\mathrm{Cr}$ (Ga)-O-Cr bond angle. To quantify the $\mathrm{CrO}_{6}$ distortion, we calculated the angles $\theta$ and $\varphi$ of the $\mathrm{CrO}_{6}$ octahedral based on the two equations $\theta=\cos ^{-1}(a / b)$ and $\varphi=\cos ^{-1}(\sqrt{ } 2 a / c)$ respectively, ${ }^{27}$ listed in Table 1 . Angle $\theta$ represents the rotation of $\mathrm{CrO}_{6}$ octahedral about (001) axis and angel $\varphi$ stands for the octahedral tilting about (110) axis. The values of both $\theta$ and $\varphi$ have an upward trend with greater doping concentration $x$, shown in Fig. 1(e) and (f), which provides an evidence of the enhancement of lattice distortion.

Fig. 2 shows the room temperature Raman spectra of $\mathrm{Sm}_{0.7} \mathrm{Y}_{0.3} \mathrm{Cr}_{1-x} \mathrm{Ga}_{x} \mathrm{O}_{3}(x=0,0.1,0.2$ and 0.3$)$ samples. According to group theory, $\mathrm{RCrO}_{3}$ with an orthorhombic $(\mathrm{Pbnm})$ has 24 types of Raman-active modes $\left(7 \mathrm{~A}_{\mathrm{g}}+5 \mathrm{~B}_{1 \mathrm{~g}}+7 \mathrm{~B}_{2 \mathrm{~g}}+5 \mathrm{~B}_{3 \mathrm{~g}}\right) \cdot{ }^{28}$ For $\mathrm{SmCrO}_{3}$, excluding some low-intensity modes, 11 modes $\left(5 \mathrm{~A}_{\mathrm{g}}+\right.$ $2 \mathrm{~B}_{1 \mathrm{~g}}+2 \mathrm{~B}_{2 \mathrm{~g}}+2 \mathrm{~B}_{3 \mathrm{~g}}$ ) are detected, which are consistent with reported Raman spectra of $\mathrm{SmCrO}_{3} \cdot{ }^{29-31}$ For only $30 \%$ Ga-doped $\mathrm{SmCrO}_{3}$, the shape and position of Raman spectra show inconspicuous changes compared to $\mathrm{SmCrO}_{3}$. Nonetheless, for $\mathrm{Sm}_{0.7} \mathrm{Y}_{0.3} \mathrm{CrO}_{3}$ sample, the obvious peaks shift of Raman spectra relative to the parent $\mathrm{SmCrO}_{3}$ occurs, which may be related to the prominent structural variations by $\mathrm{Y}$ doping, reflected and discussed in the previous work. ${ }^{14}$ For the Y- and Ga-codoped samples, the Raman spectra are almost the same to that of $\mathrm{Sm}_{0.7} \mathrm{Y}_{0.3} \mathrm{CrO}_{3}$ sample. This indicates that due to the size effect and the heavier atom of $\mathrm{Y}$ than the transitional metal ions
Raman modes are led to the obvious shift. Compared to the Raman modes and band position of $\mathrm{YCrO}_{3}$ and $\mathrm{SmCrO}_{3},{ }^{29}$ we assigned the phonon modes of $\mathrm{Sm}_{0.7} \mathrm{Y}_{0.3} \mathrm{Cr}_{1-x} \mathrm{Ga}_{x} \mathrm{O}_{3}$ samples, shown in Fig. 2. As is known that the phonon modes in $\mathrm{RCrO}_{3}$ below $200 \mathrm{~cm}^{-1}$ are related to lattice modes involving $\mathrm{R}$ atom vibrations and modes above $200 \mathrm{~cm}^{-1}$ consist of various modes involving vibrations of the $\mathrm{R}$ atom and oxygen. ${ }^{29-31}$ Specifically speaking: (1) $\mathrm{A}_{\mathrm{g}}(3)$ and $\mathrm{A}_{\mathrm{g}}(5)$ are $\mathrm{CrO}_{6}$ octahedral rotations around the crystallographic $y$-axis and $x$-axis (Pbnm setting), respectively; (2) $\mathrm{B}_{1 \mathrm{~g}}(2), \mathrm{B}_{2 \mathrm{~g}}(2)$ and $\mathrm{B}_{3 \mathrm{~g}}(1)$ are related to $\mathrm{R}$ atomic motions; (3) $\mathrm{A}_{g}(6)$ and $\mathrm{B}_{2 g}(3)$ arise from bending of the $\mathrm{CrO}_{6}$ octahedral; (4) the $\mathrm{B}_{3 \mathrm{~g}}(3)$ mode is related to the antisymmetric stretching vibrations of the $\mathrm{O}_{1}$ and $\mathrm{O}_{2}$ atoms. ${ }^{29}$ For $\mathrm{Sm}_{0.7} \mathrm{Y}_{0.3^{-}}$ $\mathrm{Ga}_{x} \mathrm{Cr}_{1-x} \mathrm{O}_{3}(x=0.1,0.2$ and 0.3$)$ samples, it indicated that Ga-

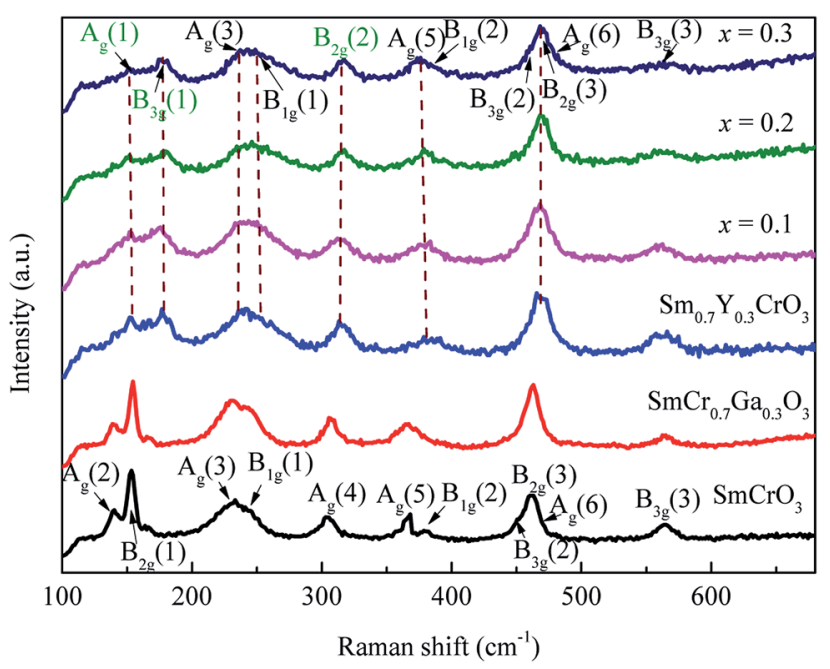

Fig. 2 The room temperature Raman spectra of samples $\mathrm{SmCrO}_{3}$, $\mathrm{SmCr}_{0.7} \mathrm{Ga}_{0.3} \mathrm{O}_{3}, \mathrm{Sm}_{0.7} \mathrm{Y}_{0.3} \mathrm{Cr}_{1-x} \mathrm{Ga}_{x} \mathrm{O}_{3}(x=0,0.1,0.2$, and 0.3$)$. 
doping does not affect the symmetric structure of the crystal. As doping concentration increases, $A_{g}(5), B_{3 g}(1)$ and $B_{2 g}(2)$ modes have a different degree of offset, in which $A_{g}(5)$ shifts to lower wavenumber (red shift) while $B_{3 g}(1)$ and $B_{2 g}(2)$ are blue shift. Red shift of $\mathrm{A}_{\mathrm{g}}(5)$ indicates the rotation of the $\mathrm{CrO}_{6}$ octahedron increases, which is consistent with the calculated $\varphi$ angle from XRD. The anomalous hardening of $B_{3 g}(1)$ and $B_{2 g}(2)$ modes reveals the possible displacement of $\mathrm{R}$ ions induced by spinphonon coupling, ${ }^{30}$ which may be related to the magnetic interactions between $\mathrm{R}^{3+}$ and $\mathrm{Cr}^{3+}$ ions., ${ }^{\mathbf{4} 30}$

The FC and ZFC curves of temperature dependent magnetization of $\mathrm{Sm}_{0.7} \mathrm{Y}_{0.3} \mathrm{Cr}_{1-x} \mathrm{Ga}_{x} \mathrm{O}_{3}(x=0.1,0.2$, and 0.3$)$ samples are shown in Fig. 3(a-c). From these graphs, it is evident that there indeed and still is antiferromagnetic transition, the SR transition, and the TIMR effect in the ZFC case. As the temperature decreases from high temperature, the magnetization curves undergo a paramagnetic to antiferromagnetic phase transition at $T_{\mathrm{N}}$. And the Neel temperature occurs below the bifurcation temperature $\left(T_{\mathrm{bf}}\right)$ of ZFC and FC curves, the value of which is around $194 \mathrm{~K}$ and is independent of doping concentration. However, the value of $T_{\mathrm{N}}$ has strongly dependence on the doping concentration, which was obtained from the maximum position of the first derivative of ZFC curves and listed in Table 1. It almost decreases linearly with a tolerance of $24 \mathrm{~K}$ with $10 \%$ doping, which results from the dilution effect of Ga ions doping on destroying the $\mathrm{Cr}-\mathrm{O}-\mathrm{Cr}$ magnetic coupling. This leads to the fact that temperature difference between $T_{\mathrm{bf}}$ and $T_{\mathrm{N}}$ becomes bigger with increase of doping concentration. The bifurcation of ZFC and FC is the characteristic of the onset of the antiferromagnetic ordering, which is attributed to the coexistence of ferromagnetic and antiferromagnetic phases caused by magnetic anisotropy. ${ }^{32-34}$ The destruction of $\mathrm{Cr}-\mathrm{O}-\mathrm{Cr}$ by the $\mathrm{Ga}^{3+}$ ions suppresses the formation of $\mathrm{Cr}^{3+}$ antiferromagnetic ordering and gives rise to the existence of ferromagnetic-like clusters, which can be demonstrated from the deviation of Curie-Weiss linear behaviour in the inverse susceptibility as a function of temperature (shown in Fig. 3(d)). Thus, an unchanged $T_{\mathrm{bf}}$ and reduced $T_{\mathrm{N}}$ are observed.

For the paramagnetic region, it was best fitted by the CurieWeiss law, $\chi=C /(T-\Theta)$, where $C$ is Curie constant and $\Theta$ is the Weiss temperature, as shown in Fig. 3(d). Based on the fitted Curie constant, the average effective magnetic moment was calculated by the following formula:

$$
\mu_{\mathrm{eff}}=\sqrt{\frac{3 k_{\mathrm{B}} C}{N}}
$$

where $k_{\mathrm{B}}$ is Boltzmann constant, $N_{\mathrm{A}}$ is Avogadro constant. All the fitted and calculated values of the parameters are summarized in Table 1. Theoretically, the whole effective magnetic (a)

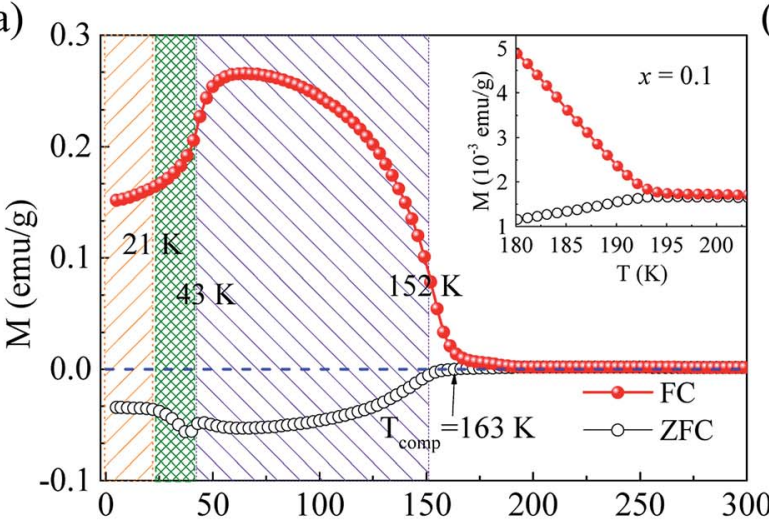

(c)

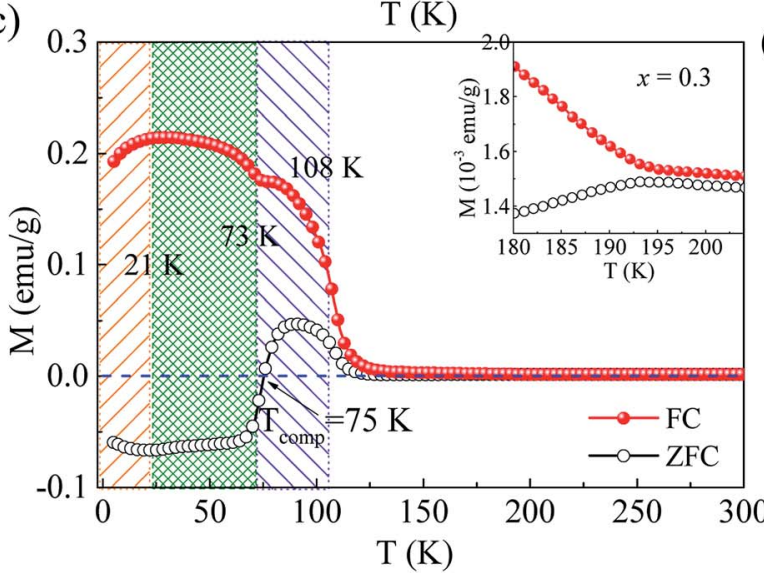

(b)

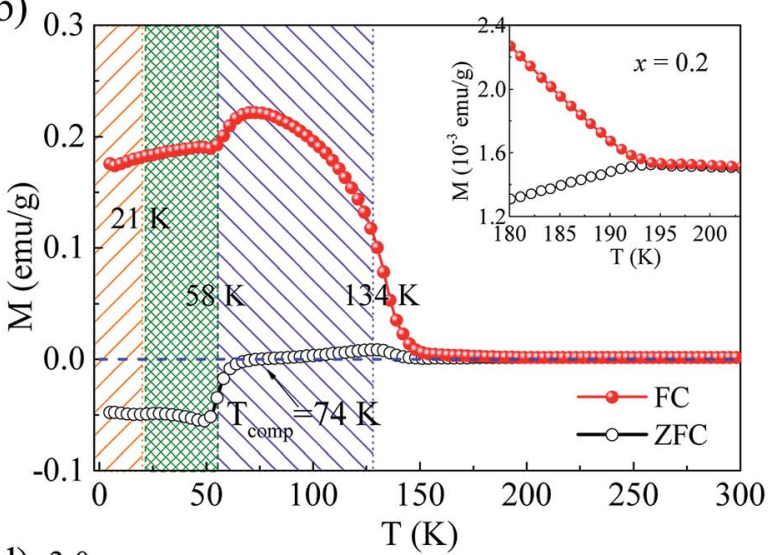

(d) 3.0

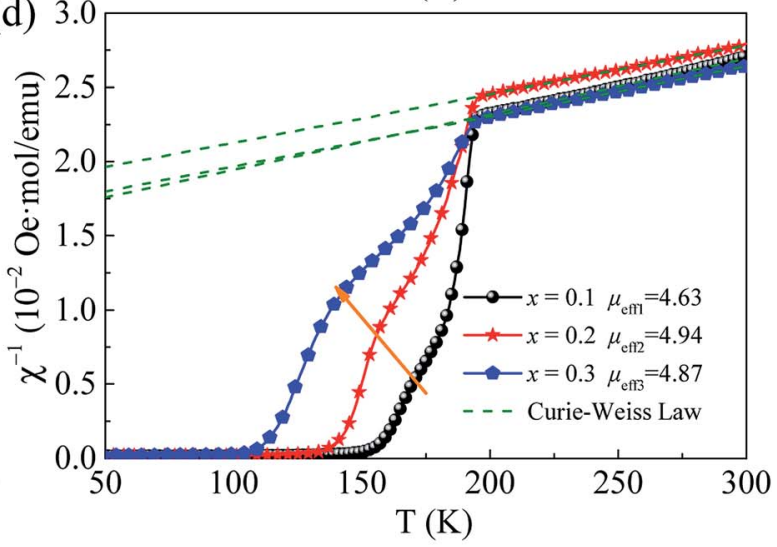

Fig. $3(\mathrm{a}-\mathrm{c})$ The ZFC and FC curves of $\mathrm{Sm}_{0.7} \mathrm{Y}_{0.3} \mathrm{Cr}_{1-x} \mathrm{Ga}_{x} \mathrm{O}_{3}(x=0.1,0.2$, and 0.3$)$. The insets show the enlarged parts of temperature near $190 \mathrm{~K}$. (d) The inverse susceptibility as a function of temperature of $x=0.1,0.2$, and 0.3 . The dot line stand for the fitting results by Curie-Weiss law. 
moment $\mu_{\text {eff }}^{*}$ in paramagnetic region can be calculated using the free ionic moments of $\mathrm{Sm}^{3+}$ and $\mathrm{Cr}^{3+}$ as the following equation

$$
\mu_{\mathrm{eff}}^{*}=\sqrt{0.7 \times \mu_{\mathrm{Sm}}{ }^{2}+0.3 \times \mu_{\mathrm{Y}}^{2}+(1-x) \mu_{\mathrm{Cr}}{ }^{2}+x \mu_{\mathrm{Ga}}{ }^{2}}
$$

where $\mu_{\mathrm{Sm}}=0.84 \mu_{\mathrm{B}}, \mu_{\mathrm{Cr}}=3.87 \mu_{\mathrm{B}}, \mu_{\mathrm{Y}}=0$, and $\mu_{\mathrm{Ga}}=0$. Note from Table 1 that the fitted effective magnetic moments ( $\left.\mu_{\text {eff }}\right)$ is around 1.3 times larger than the theoretical ones $\left(\mu_{\text {eff }}^{*}\right)$, which demonstrates that the effective magnetic moments of samples are not only from the free ionic moments of $\mathrm{Sm}^{3+}$ and $\mathrm{Cr}^{3+}$. This large calculated effective paramagnetic moment in $\mathrm{SmCrO}_{3}$ seems to be a common thing that most of the reported effective paramagnetic moment in $\mathrm{SmCrO}_{3}$ is larger than the theoretical value calculated by free magnetic ions only. ${ }^{6,16,17,35,36}$ However, few of them discuss its origin. One of the work done by ab initio calculation suggested that the pressure produced by the tilting of the oxygen octahedral causes the difference between the experimental calculated effective paramagnetic moment and the theoretical one. ${ }^{37}$ The experimental and theoretical investigations of this issue are appealed.

As temperature continues to decrease, a canted antiferromagnetic phase with weak ferromagnetic component is formed between chromium ions, showing a dramatic increment of the macroscopic magnetic moment. In the ZFC curve, an obvious negative magnetization occurs below $T_{\mathrm{N}}$ at $x=0$ (shown in our previous work $)^{14}$ and $x=0.1$. Moreover, note that when $x \geq 0.2$, the magnetization in ZFC mode is still positive when temperature is below $T_{\mathrm{bf}}$ and goes across the zero-magnetization-line to a negative value at the compensation temperature $\left(T_{\text {comp }}\right)$, showing the magnetization reversal. And this TIMR effect becomes remarkable when $x=0.3$, showing its evolution with doping concentration. Most reported TIMR effect from positive to negative occurs in FC mode while few in ZFC mode., ${ }^{2,10,25,38-42}$ Here, the TIMR effect observed in ZFC mode shows dependence on doping concentration and the compensation temperature for $x=0.2$ and 0.3 is at near $74 \mathrm{~K}$ and almost the same, indicating its intrinsic feature.

In order to prove the intrinsic nature of TIMR effect in ZFC by ruled out the effect of a small negative trapped field in the superconducting magnet during cooling on the negative magnetization, ${ }^{43}$ we measured the $\mathrm{FC} \mathrm{M}(\mathrm{T})$ curves with very small cooling fields $H_{\text {cooling }}= \pm 10$ Oe respectively and the applied field $H$ still keeps 100 Oe. The results shown in Fig. 4 show that the TIMR effect still exists both in positive and negative cooling field. This signifies that even though there is a small trapped field when doing ZFC measurement, the negative magnetization and magnetization reversal observed in our measurement would not be affected. Moreover, the TIMR effect here is actually dependent on the applied field. The fielddependence of magnetization reversal is observed, as shown in Fig. 4(b). The ZFC and FC curves under applied small field $H=$ 10 Oe were measured. It shows that magnetizations below $T_{\mathrm{N}}$ are negative in both ZFC and FC curves. And when the applied field increases upto 100 Oe the magnetization in the temperature interval $T_{\text {comp }}<T<T_{\mathrm{N}}$ becomes positive. These results prove the intrinsic nature of the TIMR effect.
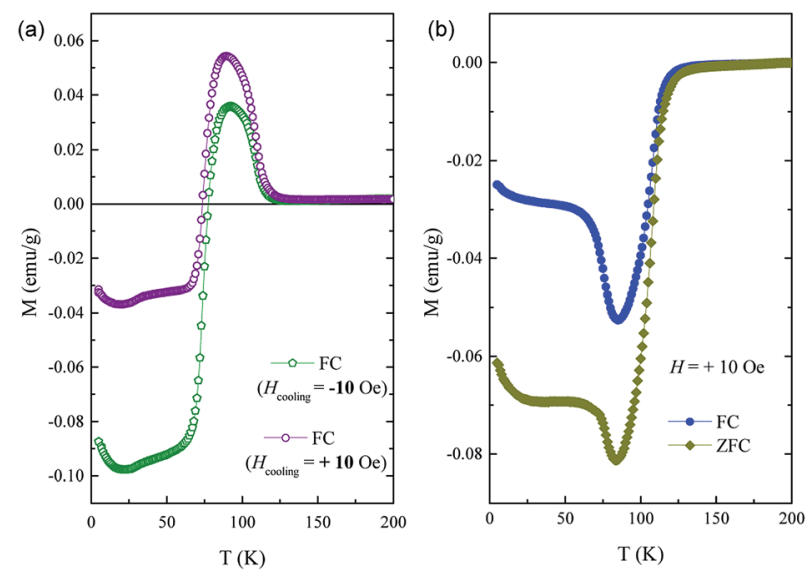

Fig. 4 (a) The field-cooling $M(T)$ curves of $\mathrm{Sm}_{0.7} \mathrm{Y}_{0.3} \mathrm{Cr}_{0.7} \mathrm{Ga}_{0.3} \mathrm{O}_{3}$ sample with cooling fields $H_{\text {cooling }}= \pm 10$ Oe respectively and the applied field is 100 Oe. (b) The ZFC and FC curves of $\mathrm{Sm}_{0.7} \mathrm{Y}_{0.3} \mathrm{Cr}_{0.7^{-}}$ $\mathrm{Ga}_{0.3} \mathrm{O}_{3}$ sample with applied field $10 \mathrm{Oe}$.

With temperature further cooling, magnetization drops abruptly in FC at $T_{\mathrm{SR}}$ for $x=0.1$ and 0.2 while rises for $x=0.3$, where the SR effect happens. In the meantime, there is a fluctuation of magnetization in ZFC at $x=0.1$ and obvious drops at $x=0.2$ and 0.3 while it never happened in $\mathrm{SmCrO}_{3}$.

This SR effect is assigned to the transition from spin configuration $\Gamma_{4}$ to $\Gamma_{2}{ }^{4,6}$ In our previous work, $\mathrm{Y}^{3+}$ doping at Sm-site has a tiny effect on the value of $T_{\mathrm{SR}}$ with $6 \mathrm{~K}$ lower at $30 \% \mathrm{Y}^{3+}$ doing sample than $\mathrm{SmCrO}_{3}{ }^{14}$ Interestingly, in the series of Ga-doped $\mathrm{Sm}_{0.7} \mathrm{Y}_{0.3} \mathrm{CrO}_{3}$ samples here, the $T_{\mathrm{SR}}$ increases uniformly with an increment of $15 \mathrm{~K}$ and reaches to 73 $\mathrm{K}$ at $x=0.3$, closely to the compensation temperature $(75 \mathrm{~K})$ and the liquid nitrogen temperature. Nonetheless, this inconsistency of $T_{\mathrm{SR}}$ and $T_{\text {comp }}$ illustrates that the TIMR effect is independent of the SR effect and the TIMR occurs ahead of the SR effect with decreasing of temperature. Moreover, the magnetization of FC curve at $\Gamma_{2}$ increases while the one at $\Gamma_{4}$ decreases correspondingly with increasing of doping concentration. As $x$ $=0.3$, the magnetization at $\Gamma_{2}$ overcomes the one at $\Gamma_{4}$. It is reasonable because that the SR transition temperature is improved to $73 \mathrm{~K}$ where the contribution of $\mathrm{Sm}^{3+}$ moments is low. And the spontaneous moment parallel to $a$ crystallographic axis $\left(\Gamma_{2}\right)$ of $\mathrm{SmCrO}_{3}$ is larger than the one parallel to $c$ crystallographic axis $\left(\Gamma_{4}\right)^{4,19}$ Moreover, the occurrence of SR transition has been attributed to the strong antisymmetric exchange interaction between $\mathrm{Sm}^{3+}$ and $\mathrm{Cr}^{3+}$ and the dilution effect of $\mathrm{Ga}$ ions doping on $\mathrm{Cr}$ ions moment gives rise to the improvement of the whole net magnetization value. With further doping, the competitiveness of $\mathrm{Cr}^{3+}$ moment relative to $\mathrm{Sm}^{3+}$ moment decreases and it will reduce and even destroy the antisymmetric $\mathrm{Sm}-\mathrm{Cr}$ interactions, leading to the parallel orientation of Sm and $\mathrm{Cr}$ spin moments, especially for the samples with high doping concentration. An enhancement of magnetization at low temperature thus will be reasonable caused for $x=0.3$.

Now we turn to give the interpretation of the TIMR accompanied with SR effect in ZFC curve. In these oxides magnetization reversal was explained in terms of competition between 
single-ion magnetic anisotropy and antisymmetric DM interactions. ${ }^{\mathbf{4 4 - 4 6}}$ In antiferromagnetic materials with low symmetry, the appearance of weak ferromagnetism is predominantly determined by either single-ion magnetocrystalline anisotropy or DM interactions. It has been suggested that the net moment produced by these mechanisms can be oriented in opposite direction and have different temperature dependence in some cases. ${ }^{47,48}$ The magnetic exchange interactions between magnetic ions generally include the isotropic, the antisymmetric (or DM interaction), and anisotropic symmetric superexchange interactions. Therefore, the Hamiltonian of the $\mathrm{SmCrO}_{3}$ system in the absence of external magnetic field can be written as follows:

$$
\begin{gathered}
H=H^{\mathrm{Cr}-\mathrm{Cr}}+H^{\mathrm{Cr}-\mathrm{Sm}} \\
H^{\mathrm{Cr}-\mathrm{Cr}}=H_{\mathrm{iso}}^{\mathrm{M}}+H_{\mathrm{anti}}^{\mathrm{M}}+H_{\text {sin }}^{\mathrm{M}} \\
H^{\mathrm{Cr}-\mathrm{Sm}}=H_{\mathrm{iso}}^{\mathrm{RM}}+H_{\mathrm{anti}}^{\mathrm{RM}}
\end{gathered}
$$

where $H_{\text {iso }}, H_{\text {anti }}$ represent the isotropic, antisymmetric exchange interactions, respectively, and $H_{\text {sin }}$ is the single-ion anisotropic term. Based on this model, we schematically plotted the spin evolution with temperature in the ZFC mode in Fig. 5 and discussed the mechanisms behind in detail in the following.

When $x \leq 0.1$ and $T<T_{\mathrm{bf}}$, the strong isotropic exchange interaction between $\mathrm{Cr}^{3+}$ spins leads to the antiferromagnetic ordering at $T_{\mathrm{N}}$. The net moment of $\mathrm{Cr}$ is oppositely aligned to
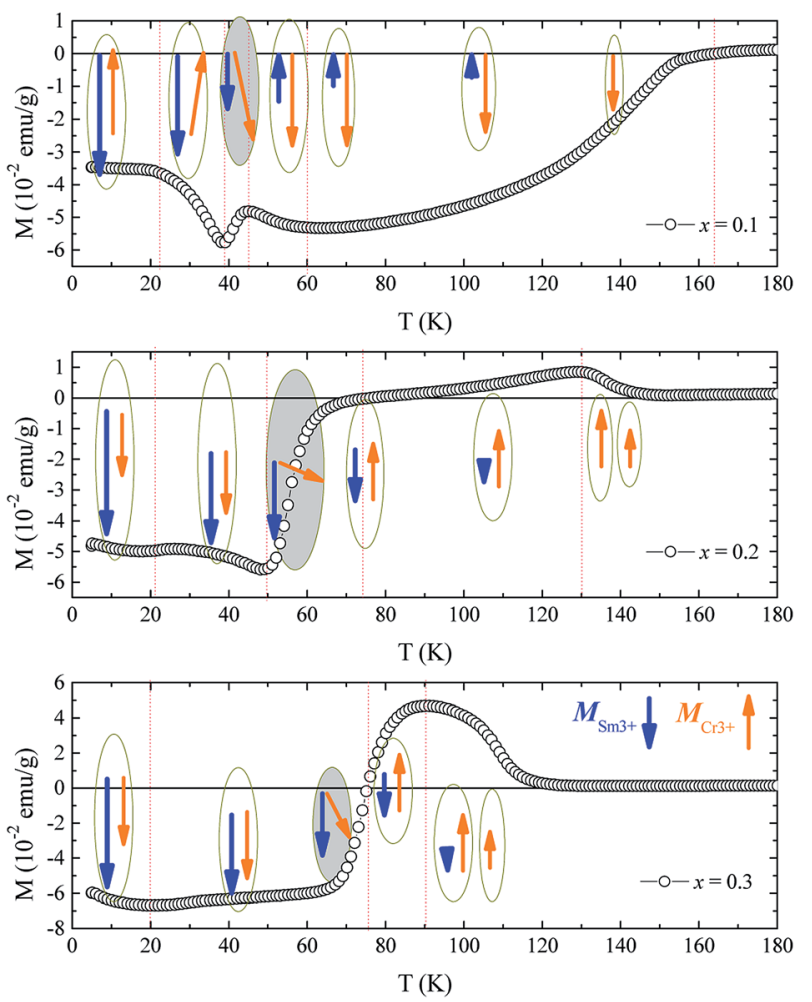

Fig. 5 The ZFC curves of samples $x=0.1,0.2$, and 0.3 and their schematic of spin evolution with temperature. the measuring field, showing the negative magnetization and increases in the absolute value with further cooling. However, for $x \geq 0.2$, the canted antiferromagnetic moments of $\mathrm{Cr}^{3+}$ spins are positive. The small canted antiferromagnetism of $\mathrm{Cr}^{3+}$ spins below $T_{\mathrm{N}}$ are attributed to two mechanisms, i.e., the single-ion anisotropy of $\mathrm{Cr}^{3+}$ ions and the antisymmetric $\mathrm{Cr}^{3+}-\mathrm{Cr}^{3+}$ exchange interactions, which do not need to have the same sign. ${ }^{48}$ Due to the dilution of $\mathrm{Cr}-\mathrm{Cr}$ exchange interaction by Ga doping, the single ion anisotropy of $\mathrm{Cr}^{3+}$ ions dominates, leading to the net moment parallel to measuring field. With further cooling, for $x=0.1$, the SR happens at $43 \mathrm{~K}$, making the spin of $\mathrm{Cr}$ deflect. At this time, the DM interaction between $\mathrm{Cr}$ spins, which has an opposite sign with that of single-ion anisotropy of $\mathrm{Cr}^{3+}$, increases and competes with the interaction of single-ion anisotropy of $\mathrm{Cr}^{3+}$. When the DM interaction between $\mathrm{Cr}-\mathrm{Cr}$ dominates over single-ion anisotropy of $\mathrm{Cr}^{3+}$, the net moment of Cr reverses and correspondingly the $\mathrm{Sm}^{3+}$ spin will also reverse due to isotropic and antisymmetric of Sm-Cr interaction. This process is accompanied by the SR effect making it happened step by step. At lower temperature, the antisymmetric $\mathrm{Sm}-\mathrm{Cr}$ interaction that produces an effective field on Cr spins becomes increasingly predominant due to the increase of Sm moment. When the effective field with respect to this antisymmetric interaction is stronger than single-ion anisotropy of $\mathrm{Cr}$ ions and the antisymmetric interaction between Cr spins below $T_{\mathrm{SR}}$, the Cr spins will rotate from the $c$ axis to $a$ axis.

With regard to samples for $x \geq 0.2$, the magnetization reverses firstly due to the antisymmetric $\mathrm{Sm}-\mathrm{Cr}$ interaction. When the moment of Sm equals to that of $\mathrm{Cr}$, the net moment becomes zero at $T_{\text {comp. }}$ Then the SR effect occurs, causing the dramatic drop of the magnetization in ZFC. If considering the isotropic and antisymmetric $\mathrm{Sm}-\mathrm{Cr}$ interactions, the spin reversal of $\mathrm{Cr}$ will be accompanied with the reversal of $\mathrm{Sm}$ moment, leading to a fluctuation of the magnetization even positive one instead of a dramatic dropping. Here the drop of the magnetization means that the isotropic and antisymmetric $\mathrm{Sm}-\mathrm{Cr}$ interactions are seriously reduced with $\mathrm{Ga}$ doping, making the magnetocrystalline anisotropy dominate. This is consistent with the reported result ${ }^{21}$ and results in the parallel arrangement of $\mathrm{Sm}^{3+}$ moment with $\mathrm{Cr}^{3+}$ net moment. Carefully seen from the ZFC curves that there is another transition at nearly $20 \mathrm{~K}$, which is obvious at $x=0.1$ and displays a broad peak at $x=0.2$ and 0.3 . This is attributed to the $\mathrm{Sm}^{3+}$ magnetic ordering with the stabilized $\mathrm{Cr}^{3+}$ spin of $\Gamma_{2}$ configuration.

Besides the TIMR and SR effect, the ZEB effect of these samples are also experimentally observed. For $\mathrm{Sm}_{0.7} \mathrm{Y}_{0.3} \mathrm{CrO}_{3}$, it was reported to show the negative ZEB effect at low temperature and reversal to positive one at $T_{\mathrm{SR}}<T<T_{\mathrm{N}}$, similar to the behaviour of $\mathrm{SmCrO}_{3}{ }^{14}$. The p-type $M-H$ curves $\left(0 \rightarrow\left(+H_{\text {max }}\right) \rightarrow\right.$ $\left.\left(-\mathrm{H}_{\max }\right) \rightarrow 0\right)$ measured under $\mathrm{ZFC}$ mode at various temperatures are shown in Fig. 6. All the curves show hysteresis loops with certain amount of coercivity $\left(H_{\mathrm{C}}\right)$ but are not saturated at high magnetic field, indicating the coexistence of weak ferromagnetism and antiferromagnetism. As is known that an EB effect is usually formed at the ferromagnetic/antiferromagnetic interface. The EB field $H_{\mathrm{EB}}$ was determined by $H_{\mathrm{EB}}=\left(H_{\mathrm{C}+}+\right.$ $\left.H_{\mathrm{C}-}\right) / 2$, where $H_{\mathrm{C}+}$ and $H_{\mathrm{C}-}$ are the left and right coercive fields 

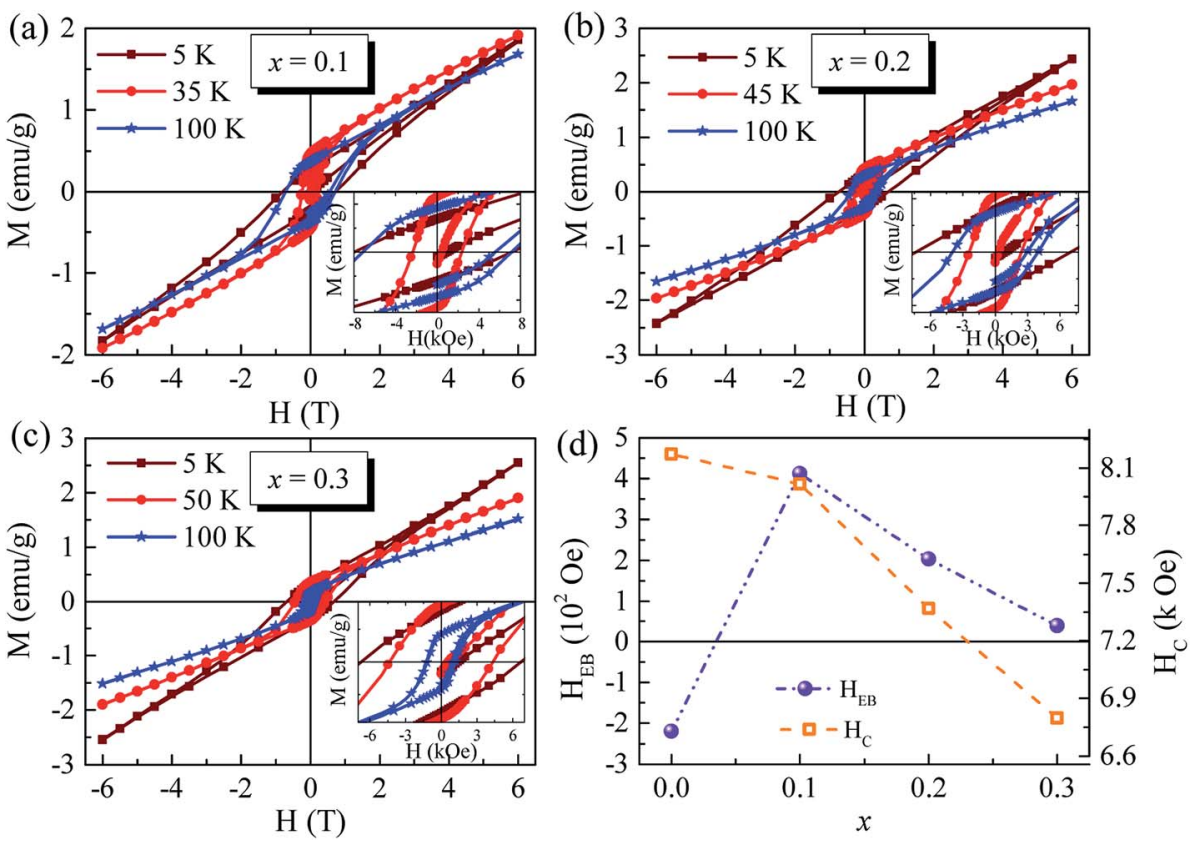

Fig. 6 (a-c) The $M-H$ curves at different temperature of samples $x=0.1,0.2$, and 0.3 . (d) The relationship of $H_{\mathrm{EB}}$ and $H_{\mathrm{C}}$ with doping concentration.

respectively. All the values of $H_{\mathrm{EB}}, H_{\mathrm{C}}$, and the remnant magnetization $M_{\mathrm{r}}$ are listed in Table 2. And the relationship of $H_{\mathrm{EB}}$ and $H_{\mathrm{C}}$ with doping concentration are plotted in Fig. 6(d). Note that at $5 \mathrm{~K}$ the ZEB effect does not only still appear but also show positive when doping $\mathrm{Ga}^{3+}$ ions and linearly decreases with increase of doping concentration. Then the ZEB effect disappears at temperature between $T_{\mathrm{N}}$ and $T_{\mathrm{SR}}$, which may be due to the stronger performance of weak ferromagnetism than antiferromagnetism. The ZEB effect was reported to be observed in the parent $\mathrm{SmCrO}_{3} \cdot{ }^{18,20}$ The EB field at $5 \mathrm{~K}$ can be reached to almost 5 kOe. ${ }^{18}$ The origin of ZEB effect in $\mathrm{SmCrO}_{3}$ is considered to be the local interaction between the canted antiferromagnetic $\mathrm{Cr}$ sublattices and orientated $\mathrm{Sm}$ sublattices. ${ }^{18}$ The coupling between $\mathrm{Sm}^{3+}$ and $\mathrm{Cr}^{3+}$ via internal magnetic field $H_{\mathrm{I}}{ }^{49}$ results in large $H_{\mathrm{EB}}{ }^{20}$ The positive ZEB effect may be related to the behaviour in layered magnetic systems, ${ }^{50,51}$ where positive EB appears if the interactions at the interfaces are antiferromagnetic, whereas negative EB is present for ferro-magnetic interactions. As reported, by nonmagnetic rare-earth ions

Table 2 Exchange bias field $H_{\mathrm{EB}}$, coercive field $H_{\mathrm{C}}$, and residual magnetization $\mathrm{M}_{\mathrm{r}}$ extracted from $\mathrm{M}-\mathrm{H}$ loops of $\mathrm{Sm}_{0.7} \mathrm{Y}_{0.3} \mathrm{Ga}_{x} \mathrm{Cr}_{1-x} \mathrm{O}_{3}$ $(x=0.1,0.2,0.3)$ samples at different temperatures

\begin{tabular}{llccl}
\hline Sample & Temperature & $H_{\text {Ев }}(\mathrm{Oe})$ & $H_{\mathrm{C}}(\mathrm{Oe})$ & $M_{\mathrm{r}}\left(\mathrm{emu} \mathrm{g}^{-1}\right)$ \\
\hline$x=0.1$ & $5 \mathrm{~K}$ & 412.6 & 8015.3 & 0.27455 \\
& $35 \mathrm{~K}$ & 5.3 & 2345.6 & 0.36946 \\
$x=0.2$ & $100 \mathrm{~K}$ & 2.5 & 7154.4 & 0.45936 \\
& $5 \mathrm{~K}$ & 202.6 & 7368.1 & 0.31416 \\
& $45 \mathrm{~K}$ & -2.8 & 2388.6 & 0.41866 \\
$x=0.3$ & $100 \mathrm{~K}$ & 7.5 & 3945.3 & 0.29665 \\
& $5 \mathrm{~K}$ & 39.85 & 6796.3 & 0.31861 \\
& $50 \mathrm{~K}$ & 10.75 & 4361.3 & 0.35168 \\
& $100 \mathrm{~K}$ & -2.1 & 1179.4 & 0.17103
\end{tabular}

doping at Sm-site, such as La, the exchange bias field $H_{\mathrm{EB}}$ decreases monotonously with doping level increasing due to the weakness of the Sm-Cr coupling. ${ }^{20}$ The $H_{\mathrm{EB}}$ of ZEB effect in our samples show the decrement as well with increase of nonmagnetic $\mathrm{Ga}^{3+}$ ions doping, similar to the reported results. ${ }^{20}$ The nonmagnetic ions doping $\left(\mathrm{Y}^{3+}\right.$, and $\left.\mathrm{Ga}^{3+}\right)$, nonmatter doping at $\mathrm{Sm}$ - or Cr-sites, would reduce the coupling between $\mathrm{Sm}^{3+}$ and $\mathrm{Cr}^{3+}$, leading to the decrease of ZEB effect.

\section{Conclusions}

In conclusion, we investigated the temperature dependent magnetic properties of $\mathrm{SmCrO}_{3}$ by codoping nonmagnetic ions at Sm- and Cr-sites. It is evident that there indeed and still exists antiferromagnetic transition, the TIMR effect, and the SR effect. The spin reorientation from $\Gamma_{4}$ to $\Gamma_{2}$ is tuned and the transition temperature $T_{\mathrm{SR}}$ is improved dramatically to the liquid nitrogen temperature by Ga ions doping, which would be helpful to achieve its application in temperature sensitive spintronic devices and magnetic switching devices. The intrinsic TIMR effect from positive to negative under ZFC condition are significantly induced as well and its reversal evolution is strongly dependent with doping. Moreover, the positive ZEB effect is formed in Ga doped $\mathrm{Sm}_{0.7} \mathrm{Y}_{0.3} \mathrm{CrO}_{3}$ samples at $5 \mathrm{~K}$ although it is suppressed with increase of doping concentration and disappears in high temperature. Under the influence of doping nonmagnetic ions, lattice distortion is induced to some extend and the magnetic interactions of $\mathrm{Cr}-\mathrm{Cr}$ and $\mathrm{Sm}-\mathrm{Cr}$ are predominantly diluted, leading to tune the above phenomena. Those phenomena are discussed and successfully explained by considering the magnetic exchange interactions competitions including the isotropic, antisymmetric (or DM interaction), and anisotropic superexchange interactions. 


\section{Conflicts of interest}

There are no conflicts to declare.

\section{Acknowledgements}

This work was supported by the National Natural Science Foundation of China (Grant No. 11605092 and 11704009) and Nanjing University of Posts and Telecommunications under research project (No. NY215091).

\section{References}

1 J. R. Sahu, C. R. Serrao and C. N. R. Rao, Solid State Commun., 2008, 145, 52-55.

2 Y. Su, J. Zhang, Z. Feng, L. Li, B. Li, Y. Zhou, Z. Chen and S. Cao, J. Appl. Phys., 2010, 108, 013905.

3 J. S. Zhou, J. A. Alonso, V. Pomjakushin, J. B. Goodenough, Y. Ren, J. Q. Yan and J. G. Cheng, Phys. Rev. B, 2010, 81, 214115.

4 B. Rajeswaran, D. I. Khomskii, A. K. Zvezdin, C. N. R. Rao and A. Sundaresan, Phys. Rev. B, 2012, 86, 214409.

5 Y. Cao, S. Cao, W. Ren, Z. Feng, S. Yuan, B. Kang, B. Lu and J. Zhang, Appl. Phys. Lett., 2014, 104, 232405.

6 S. Huang, G. Zerihun, Z. Tian, S. Yuan, G. Gong, C. Yin and L. Wang, Ceram. Int., 2014, 40, 13937-13943.

7 A. McDannald, C. R. dela Cruz, M. S. Seehra and M. Jain, Phys. Rev. B, 2016, 93, 184430.

8 E. F. Bertaut, G. Bassi, G. Buisson, P. Burlet, J. Chappert, A. Delapalme, J. Mareschal, G. Roult, R. Aleonard, R. Pauthenet and J. P. Rebouillat, J. Appl. Phys., 1966, 37, 1038-1039.

9 A. Ghosh, K. Dey, M. Chakraborty, S. Majumdar and S. Giri, EPL, 2014, 107, 47012.

10 L. H. Yin, Y. Liu, S. G. Tan, B. C. Zhao, J. M. Dai, W. H. Song and Y. P. Sun, Mater. Res. Bull., 2013, 48, 4016-4021.

11 X. L. Qian, D. M. Deng, Y. Jin, B. Lu, S. X. Cao and J. C. Zhang, J. Appl. Phys., 2014, 115, 193902.

12 M. Tripathi, R. J. Choudhary and D. M. Phase, RSC Adv., 2016, 6, 90255-90262.

13 Y. Wu, J. Xu and Z. Xia, J. Low Temp. Phys., 2016, 183, 14-22.

14 H. Zhang, J. Wang, L. Xie, D. Fu, Y. Guo and Y. Li, J. Appl. Phys., 2017, 122, 204103.

15 D.-x. Fu, Y.-z. Liu, H.-g. Zhang, L. Xie and B. Li, J. Alloys Compd., 2018, 735, 1052-1062.

16 S. Huang, K. P. Su, H. O. Wang, L. R. Shi and D. X. Huo, Ceram. Int., 2017, 43, 12258-12262.

17 X. Qian, L. Chen, S. Cao and J. Zhang, Solid State Commun., 2014, 195, 21-25.

18 P. Gupta, R. Bhargava and P. Poddar, J. Phys. D: Appl. Phys., 2015, 48, 025004.

19 G. Gorodetsky, R. M. Hornreich, S. Shaft, B. Sharon, A. Shaulov and B. M. Wanklyn, Phys. Rev. B, 1977, 16, 515521.

20 S. Huang, L. R. Shi, Z. M. Tian, H. G. Sun and S. L. Yuan, J. Magn. Magn. Mater., 2015, 394, 77-81.
21 M. Tripathi, R. J. Choudhary, D. M. Phase, T. Chatterji and H. E. Fischer, Phys. Rev. B, 2017, 96, 174421.

22 Y. Fang, X. Cui, J. Kang, W. Sun, P. Cheng, F. Chen and J. Zhang, Solid State Commun., 2017, 261, 37-40.

23 J. Kang, Y. Yang, X. Qian, K. Xu, X. Cui, Y. Fang, V. Chandragiri, B. Kang, B. Chen, A. Stroppa, S. Cao, J. Zhang and W. Ren, IUCrJ, 2017, 4, 598-603.

24 Y. Fang, S.-M. Yan, Y.-Y. Gong, W.-L. Zhu, Q.-Q. Cao, D.-H. Wang and Y.-W. Du, Chin. Phys. B, 2014, 23, 127502.

25 N. Panwar, J. P. Joby, S. Kumar, I. Coondoo, M. Vasundhara, N. Kumar, R. Palai, R. Singhal and R. S. Katiyar, AIP Adv., 2018, 8, 055818.

26 B. H. Toby, J. Appl. Crystallogr., 2001, 34, 210-213.

27 D. J. W. Yusheng Zhao, J. B. Parise and D. E. Cox, Phys. Earth Planet. Inter., 1993, 76, 16.

28 M. N. Iliev, M. V. Abrashev, H. G. Lee, V. N. Popov, Y. Y. Sun, C. Thomsen, R. L. Meng and C. W. Chu, Phys. Rev. B, 1998, 57, 2872-2877.

29 M. C. Weber, J. Kreisel, P. A. Thomas, M. Newton, K. Sardar and R. I. Walton, Phys. Rev. B, 2012, 85, 054303.

30 V. Srinu Bhadram, B. Rajeswaran, A. Sundaresan and C. Narayana, EPL, 2013, 101, 17008.

31 V. S. Bhadram, D. Swain, R. Dhanya, M. Polentarutti, A. Sundaresan and C. Narayana, Mater. Res. Express, 2014, 1, 026111.

32 T. Krenke, M. Acet, E. F. Wassermann, X. Moya, L. Mañosa and A. Planes, Phys. Rev. B, 2005, 72.

33 R. Das, A. Jaiswal, S. Adyanthaya and P. Poddar, J. Appl. Phys., 2011, 109, 064309.

34 S. Lei, L. Liu, C. Wang, C. Wang, D. Guo, S. Zeng, B. Cheng, Y. Xiao and L. Zhou, J. Mater. Chem. A, 2013, 1, 11982.

35 Z. Xiang, W. Li and Y. Cui, RSC Adv., 2018, 8, 8842-8848.

36 L. M. Daniels, M. C. Weber, M. R. Lees, M. Guennou, R. J. Kashtiban, J. Sloan, J. Kreisel and R. I. Walton, Inorg. Chem., 2013, 52, 12161-12169.

37 H. J. Zhao, W. Ren, X. M. Chen and L. Bellaiche, J. Phys.: Condens. Matter, 2013, 25, 385604.

38 T. Bora and S. Ravi, J. Magn. Magn. Mater., 2014, 358-359, 208-211.

39 P. Gupta and P. Poddar, Inorg. Chem., 2015, 54, 9509-9516. 40 L. Wang, G. H. Rao, X. Zhang, L. L. Zhang, S. W. Wang and Q. R. Yao, Ceram. Int., 2016, 42, 10171-10174.

41 L. Wang, S. W. Wang, X. Zhang, L. L. Zhang, R. Yao and G. H. Rao, J. Alloys Compd., 2016, 662, 268-271.

42 S. Kumar, I. Coondoo, M. Vasundhara, A. K. Patra, A. L. Kholkin and N. Panwar, J. Appl. Phys., 2017, 121, 043907.

43 N. Kumar and A. Sundaresan, Solid State Commun., 2010, 150, 1162-1164.

44 T. Moriya, Phys. Rev., 1960, 120, 91-98.

45 I. Dzyaloshinsky, J. Phys. Chem. Solids, 1958, 4, 241-255.

46 P. Mandal, A. Sundaresan, C. N. R. Rao, A. Iyo, P. M. Shirage, Y. Tanaka, C. Simon, V. Pralong, O. I. Lebedev, V. Caignaert and B. Raveau, Phys. Rev. B, 2010, 82, 100416.

47 Y. Ren, T. T. M. Palstra, D. I. Khomskii, E. Pellegrin, A. A. Nugroho, A. A. Menovsky and G. A. Sawatzky, Nature, 1998, 396, 441. 
48 Y. Ren, T. Palstra, D. Khomskii, A. Nugroho, A. Menovsky and G. Sawatzky, Phys. Rev. B, 2000, 62, 6577-6586.

49 K. Yoshii, Appl. Phys. Lett., 2011, 99, 142501.
50 J. Nogués and I. K. Schuller, J. Magn. Magn. Mater., 1999, 192, 203-232.

51 W. Wang, F. Takano, M. Takenaka, H. Akinaga and H. Ofuchi, J. Appl. Phys., 2008, 103, 093914. 